\title{
Normal solutions for master equations with time-dependent transition rates: Application to heating processes
}

\author{
J. J. Brey and A. Prados \\ Física Teórica, Facultad de Física, Universidad de Sevilla, Apartado de Correos 1065, \\ E-41080 Sevilla, Spain \\ (Received 10 August 1992)
}

\begin{abstract}
The long-time limit of the solutions of a master equation with time-dependent transition rates is analyzed, and the existence of a special (normal) solution, that all the other solutions tend to approach, is shown under quite general conditions. In general, the normal solution will be quite different from the time-dependent equilibrium distribution, although for not too fast continuous heating processes, it asymptotically tends to the equilibrium curve. The results seem to be relevant for the explanation of what is observed in real experiments. The general theory is applied to a very simple model, for which the normal solution is exactly found.
\end{abstract}

PACS number(s): 02.50.-r, 05.70.Ln, 61.43.Bn

\section{INTRODUCTION}

The modeling of many physical processes by means of master equations requires the consideration of timedependent transition rates. This is the case, for instance, when the variation of one or more intensive thermodynamic parameters, such as the pressure or the temperature, is assumed to be externally controlled. Several models that attempt to explain the peculiar behavior shown by glasses under continuous heating or cooling have been formulated in this way [1].

While there is a well-structured theory for master equations with time-independent transition rates [2], results for the more general situation of time dependence are scarce. Nevertheless, an analysis of the literature shows that rather different models lead to similar results, and one has the feeling that the same general feature is being rederived many times. This situation is not only unpleasant from a formal point of view, but it also makes difficult the progress in our physical understanding of the phenomena we are trying to describe. We must be able to separate general behaviors from those that are specific for the model under consideration.

One of the most fundamental and celebrated properties of the master equation with constant transition rates is that, as the time goes to infinity, all solutions tend to be the equilibrium solution. This theorem holds when the Markov process defined by the master equation has a finite number of states and it is irreducible, i.e., all the configurations can be reached from a given one by a string of transitions with nonzero probability.

The first point we address in this paper is whether a generalization of the above property holds when the transition rates change in time following a given law. It will be shown that, under conditions quite similar to those considered in the constant-rate case, all solutions of the master equation converge. For cooling processes, the applicability of the result is limited by the possible freezing of the system and the loss of irreducibility. But, in other cases, there is a special solution of the master equation which all the other solutions tend to approach. We will refer to this solution as the normal solution for the given transition rates.

The generality of the above result must be stressed. It is not restricted to near-equilibrium situations or to processes in which only the variation of the tempeature is involved. Besides, given the arbitrariness of the processes for which it applies, the long-time limit does not imply that any of the physical parameters appearing in the transition rates is going to infinity. The evolution of the system can be such that all its parameters remain bounded for all times. This is the case, for instance, when periodic transition rates are considered. In this context, the normal solution for a given system is not only an asymptotic solution but is expected to be defined for the entire range of variation of the parameters. The situation is similar to the one found in kinetic theory, from which the term normal solution has been borrowed. The existence of these normal solutions is the main result of this paper.

A particularly interesting case is the continuous heating of a system. The experience shows that for usual laboratory heating rates the system always reaches the equilibrium curve at high temperatures. Of course, it is trivial to see that the time-dependent equilibrium distribution is an asymptotic solution of the master equation at infinite temperature when the system is heated slowly. We will also present here a proof that all the solutions of the master equation, including the normal one, show that asymptotic behavior in the long-time limit, under welldefined conditions.

The existence of a normal solution has serious implications for the behavior of the system that is being heated. Also, it explains the hysteresis effects that are observed when a system is cooled to low temperatures and then heated. Therefore, the construction of the normal solutoin is a main step in understanding the behavior of a system with time-dependent transition rates. This may be a difficult task for realistic models, and require the use of approximation techniques. Nevertheless, for very simple but important models the normal solution can be found 
exactly. An example will be given here.

We want to emphasize the kind of processes we will consider. The transition rates are assumed to be externally controlled, their time evolution being independent of the state of the system. In this sense, those models where the transition rates depend on time through some functional of the distribution function are excluded. Therefore, the derivations presented here apply neither to usual mean-field-model approximations nor to the case of the presence of forces controlling the state of the system (e.g, thermostat forces). The existence of normal solutions for these situations will be discussed in a separate paper.

The plan of the paper is as follows. In the next section, the long-time limit of the solutions of a master equation with time-dependent transition probabilities will be analyzed. The particular case of continuous heating processes is studied in Sec. III. Finally, in Sec. IV, the application to a two-level-system model is presented.

\section{LONG-TIME LIMIT}

Consider a system whose dynamics is described by the master equation

$$
\frac{d p_{i}}{d t}=\sum_{j}\left[W_{i j}(t) p_{j}-W_{j i}(t) p_{i}\right]
$$

Here $p_{i}(t)$ is the probability of finding the system in state $i$ at time $t$, and the transition rates $W_{i j}$ for changes from state $j$ to state $i$ depend on time in a given way. We define a quantity $H(t)$ by

$$
H(t)=\sum_{i} p_{i}(t) \ln \frac{p_{i}(t)}{p_{i}^{\prime}(t)},
$$

where $p_{i}(t)$ and $p_{i}^{\prime}(t)$ are two solutions of Eq. (1) corresponding to different initial conditions. The above definition requires that $p_{i}^{\prime}(t)$ is positive for all $i$. If the process defined by Eq. (1) is irreducible, this condition will be verified after an initial transient period, even in the case that the initial probabilities of some states were zero. The time variation of $H(t)$ is given by

$$
\frac{d H(t)}{d t}=\sum_{i} \frac{d p_{i}(t)}{d t} \ln \frac{p_{i}(t)}{p_{i}^{\prime}(t)}-\sum_{i} \frac{p_{i}(t)}{p_{i}^{\prime}(t)} \frac{d p_{i}^{\prime}(t)}{d t} .
$$

Using Eq. (1) we can transform this expression into

$$
\frac{d H(t)}{d t}=A(t)-\sum_{i} \frac{p_{i}}{p_{i}^{\prime}}\left[\frac{d p_{i}^{\prime}(t)}{d t}-\sum_{j}\left(W_{i j} p_{j}^{\prime}-W_{j i} p_{i}^{\prime}\right]\right.
$$

with

$$
\begin{aligned}
A(t)=\sum_{i, j} W_{i j} p_{j}^{\prime} & {\left[\left[\frac{p_{j}}{p_{j}^{\prime}}-\frac{p_{i}}{p_{i}^{\prime}}\right]\left[\ln \frac{p_{i}}{p_{i}^{\prime}}+1\right]\right.} \\
& \left.+\frac{p_{i}}{p_{i}^{\prime}} \ln \frac{p_{i}}{p_{i}^{\prime}}-\frac{p_{j}}{p_{j}^{\prime}} \ln \frac{p_{j}}{p_{j}^{\prime}}\right] .
\end{aligned}
$$

Up to this point, we have not used yet that $p_{i}^{\prime}(t)$ is also a solution of the master equation. We have written Eq. (4) for further reference, but in our present discussion it is clear that the second term on the right-hand side of that equation vanishes, and we get

$$
\frac{d H(t)}{d t}=A(t)
$$

It is easily seen [2] that $A(t) \leq 0$, the equality sign holding only when

$$
\frac{p_{j}(t)}{p_{j}^{\prime}(t)}=\frac{p_{i}(t)}{p_{i}^{\prime}(t)},
$$

for all pairs of states $i, j$ with $W_{i j}(t) \neq 0$. Besides, $H(t)$ is bounded below. It follows that it must tend to a limit and, therefore,

$$
\frac{p_{j}(\infty)}{p_{j}^{\prime}(\infty)}=\frac{p_{i}(\infty)}{p_{i}^{\prime}(\infty)}
$$

if $W_{i j}(\infty) \neq 0$. When the long-time limit of the transition rates still defines an irreducible process, Eq. (8) implies

$$
p_{i}(\infty)=p_{i}^{\prime}(\infty)
$$

for all $i$, i.e., all the solutions of the master equation converge toward the same behavior.

The above result does not apply when the ergodicity of the system is lost as $t \rightarrow \infty$. This excludes, in particular, the continuous cooling of a physical system to $T=0 \mathrm{~K}$, where all the transition rates are expected to vanish and the state of the system becomes frozen. This is related to the existence of residual properties obtained in models proposed to mimic the dynamic behavior of glasses at low temperatures [1]. On the other hand, models describing the continuous heating of a system or a periodic variation of the temperature will satisfy, in general, the conditions required in the proof. In these cases, Eq. (9) can be understood as showing the existence of a long-time regime, where the influence of the initial conditions has been lost, and the state of the system and its dynamics is fully determined by the law of variation of the temperature. A similar discussion can be made when the externally controlled parameter is other than the temperature.

\section{CONTINUOUS HEATING}

Let us assume that for arbitrary $t$, the equation

$$
\sum_{j} W_{i j}(t) p_{j}^{0}(t)=\sum_{j} W_{j i}(t) p_{i}^{0}(t)
$$

has the canonical solution

$$
p_{j}^{0}=\frac{1}{Z(t)} \exp \left[-\frac{\varepsilon_{j}}{k_{B} T(t)}\right] .
$$

Here $k_{B}$ is the Boltzmann constant, $T$ is the temperature, $\varepsilon_{j}$ is the energy of state $j$, and

$$
Z(t)=\sum_{j} \exp \left[-\frac{\varepsilon_{j}}{k_{B} T(t)}\right]
$$

is a time-dependent partition function. As indicated in Eqs. (11) and (12) all the time dependence of $p_{j}^{0}$ is as- 
sumed to take place through the temperature. The existence of $p_{j}^{0}$ is guaranteed by the irreducibility of the process for all times. Nevertheless, the explicit form in Eq. (11) has been assumed for the sake of concreteness, although a similar analysis can be carried out for other physical distributions, e.g., the isothermal-isobaric one.

Of course, $p_{j}^{0}(t)$ is not a solution of the master equation, due to the variation of the temperature. Define

$$
H^{0}(t)=\sum_{i} p_{i}(t) \ln \frac{p_{i}(t)}{p_{i}^{0}(t)},
$$

where, again, $p_{i}(t)$ is a solution of Eq. (1). Application of Eq. (4) yields

$$
\frac{d H^{0}(t)}{d t}=A^{0}(t)-\sum_{i} \frac{p_{i}}{p_{i}^{0}} \frac{d p_{i}^{0}}{d t},
$$

with $A^{0}(t)$ given by Eq. (5), but replacing $p_{i}^{\prime}$ by $p_{i}^{0}(t)$. Using Eq. (11) it is found

$$
\sum_{i} \frac{p_{i}}{p_{i}^{0}} \frac{d p_{i}^{0}}{d t}=\frac{1}{k_{B} T^{2}} \frac{d T}{d t}\left[\langle E(t)\rangle-\langle E(t)\rangle_{0}\right] .
$$

We use the notation

$$
\langle E(t)\rangle=\sum_{i} p_{i}(t) \varepsilon_{i}
$$

and

$$
\langle E(t)\rangle_{0}=\sum_{i} p_{i}^{0}(t) \varepsilon_{i}
$$

i.e., $\langle E(t)\rangle$ is the actual average energy at time $t$, while $\langle E(t)\rangle_{0}$ is the average energy that the system would have at equilibrium at the temperature $T(t)$.

For arbitrary laws of temperature variation little information can be obtained from Eqs. (13) and (14), since $H^{0}(t)$ does not have a well-defined sign. The initial condition can always be chosen so as to make $H^{0}(t)$ positive and, therefore, $H^{0}$ is not a monotonically decreasing quantity nor is the equilibrium distribution monotonically approached for all times. Nevertheless, if we restrict ourselves to a continuous heating of the system, the term

$$
B(t)=\frac{1}{k_{B} T^{2}} \frac{d T}{d t}\langle E(t)\rangle_{0}
$$

goes to zero under very general conditions. More precisely, if the equilibrium energy behaves as $T^{\alpha}$ in the limit of high temperatures, the term given by Eq. (18) tends to vanish if the asymptotic temperature variation rate becomes negligible compared with $T^{2-\alpha}$. For normal systems the energy is either bounded or it increases linearly with the temperature at high temperatures (constant specific heat). In the former case it is $\alpha=0$ and the condition becomes

$$
\lim _{t \rightarrow \infty} T^{-2} \frac{d T}{d t}=0
$$

while in the latter $\alpha=1$ and it must be

$$
\lim _{t \rightarrow \infty} \frac{d \ln T}{d t}=0 \text {. }
$$

All the heating processes in which the limit $T \rightarrow \infty$ requires also $t \rightarrow \infty$ satisfy Eq. (20) and, therefore, Eq. (19).

Since $B(t)$ is the only term giving a positive contribution to the variation of $H^{0}$, we arrive at

$$
\lim _{t \rightarrow \infty} \frac{d H^{0}(t)}{d t} \leq 0
$$

However, taking into account that $H^{0}(t)$ is bounded below, it follows that the only possibility is, in fact, the equality. Thus, it must be

$$
\lim _{t \rightarrow \infty} A^{0}(t)=0
$$

and

$$
\lim _{t \rightarrow \infty} \frac{1}{k_{B} T^{2}} \frac{d T}{d t}\langle E(t)\rangle=0 .
$$

As stated before, we expect the master equation to be irreducible in the limit $t \rightarrow \infty$, so Eq. (22) implies that

$$
p_{i}(\infty)=p_{i}^{0}(\infty)
$$

and Eq. (23) becomes equivalent to our assumption that $B(t)$, defined by Eq. (18), vanishes for $t \rightarrow \infty$.

Therefore, we have established that under continuous heating all solutions of Eq. (1) tend asymptotically to the time-dependent equilibrium distribution. The derivation given above requires that the process defined by the master equation is irreducible for all values of $T$, and also that the heating rate is not too large, in the sense that $B(t)$, defined by Eq. (18), must go to zero in the long-time limit.

Putting together the results of the last two sections, the following picture emerges for the time evolution of a system whose temperature is monotonically increased: Starting from an arbitrary initial condition first, the system tends to a regime where its state is determined by the heating law, while the initial condition has been forgotten. Later on, the curve describing the state of the system in this regime asymptotically approaches the temperature-dependent equilibrium curve. The main implication of this description should be the existence of a special solution of the master equation for each heating law, such that all the other solutions would approach it after an initial transient period. Following the terminology that is usual in kinetic theory, we will refer to this special solution as the normal solution of the master equation for the given heating law.

The existence of a normal solution for heating processes may play a crucial role in the explanation of the hysteresis effects that have been observed when a system is cooled and then heated. These effects have been extensively studied and analyzed in glasses [3], but the results derived here can be applied to any system.

As is the case in kinetic theory, the construction of a normal solution may be a formidable task. We have investigated the possibility of generating it by means of the Hilbert method, and the results will be presented elsewhere. In this paper we will only discuss a simple model for which the normal solution can be easily found. 


\section{NORMAL SOLUTION FOR A TWO-LEVEL SYSTEM}

The two-level system (TLS) has been used, in spite of its simplicity, as a model to study the static properties of glasses at low temperatures [4], and also their dynamical properties at high temperatures [5-7]. The TLS has only two states of energies 0 and $\varepsilon$. To jump from one state to the other, the system must be thermally activated over a barrier of energy $V$, measured from the upper state. The transition rate for hopping from the upper state over the barrier into the lower state is

$$
\gamma(t)=\alpha \exp [-\beta(t) V],
$$

where $\beta(t)=\left[k_{B} T(t)\right]^{-1}$, and $\alpha$ is the transition attempt frequency. We will use it to define an adimensional time variable, i.e., we formally take $\alpha=1$.

Since the model has only one degree of freedom, the master equation can be written as a closed equation for the probability $p(t)$ of finding the system in the upper state. Introducing the asymmetry parameter

$$
\mu=\frac{\varepsilon}{V},
$$

the equation reads

$$
\dot{p}(t)=\gamma(t)\left\{\gamma(t)^{\mu}-\left[1+\gamma(t)^{\mu}\right] p(t)\right\} .
$$

If the temperature is a monotonous function of time, and its variation is given by

$$
\dot{\gamma}(t)=r g[\gamma(t)],
$$

with $r>0$ and $g>0$, the general solution of Eq. (27) is

$$
\begin{aligned}
p(\gamma)= & p_{0} \exp \left[-\frac{1}{r} \int_{\gamma_{0}}^{\gamma} d y \frac{y+y^{1+\mu}}{g(y)}\right] \\
& +\frac{1}{r} \int_{\gamma_{0}}^{\gamma} d y \frac{y^{1+\mu}}{g(y)} \exp \left[-\frac{1}{r} \int_{y}^{\gamma} d z \frac{z+z^{1+\mu}}{g(y)}\right] .
\end{aligned}
$$

Here $p_{0}=p\left(\gamma_{0}\right)$, where $\gamma_{0}$ is the value of $\gamma$ corresponding to the initial temperature. To analyze Eq. (29) it is convenient to introduce the variable

$$
x=\frac{1}{r} \int_{0}^{\gamma} d y \frac{y+y^{1+\mu}}{g(y)},
$$

which is a strictly growing function of $x$, and verified

$$
\lim _{\gamma \rightarrow 0} x=0 \text {. }
$$

Moreover, if we consider heating laws satisfying Eq. (19), it is

$$
\lim _{\gamma \rightarrow 1} x=\infty .
$$

Notice that the limits $\gamma \rightarrow 0$ and $\gamma \rightarrow 1$ correspond to $T \rightarrow 0$ and $T \rightarrow \infty$, respectively. In terms of $x$, Eq. (29) becomes

$$
\begin{aligned}
p(x)= & p_{0} \exp \left[-\left(x-x_{0}\right)\right] \\
& +\int_{x_{0}}^{x} d x^{\prime} \phi_{0}\left(x^{\prime}\right) \exp \left[-\left(x-x^{\prime}\right)\right],
\end{aligned}
$$

where $\phi_{0}(x)$ is the equilibrium distribution of the TLS written as a function of $x$, i.e.,

$$
\phi_{0}(x)=\frac{\gamma(x)^{\mu}}{1+\gamma(x)^{\mu}} .
$$

For $x \gg x_{0}$ and $x-x_{0} \gg 1$, Eq. (33) reduces to

$$
p(x)=\int_{0}^{x} d x^{\prime} \phi_{0}\left(x^{\prime}\right) \exp \left[-\left(x-x^{\prime}\right)\right]
$$

The limits leading from Eq. (33) to Eq. (35) correspond to a long-time limit for which the initial condition is forgotten. Therefore, Eq. (35) is the normal solution whose existence was discussed in the previous section. From a formal point of view, it can also be derived from Eq. (33) by assuming that for $T=0(x=0)$ the system was at equilibrium $\left(p_{0}=0\right)$.

Integration by parts of Eq. (35) yields

$$
p(x)=\phi_{0}(x)-\int_{0}^{x} d x^{\prime} \frac{d \phi_{0}\left(x^{\prime}\right)}{d x^{\prime}} \exp \left[-\left(x-x^{\prime}\right)\right],
$$

and the condition given by Eq. (32) implies that for $x \rightarrow \infty$, the distribution tends to the equilibrium one. It must be realized that, even if the heating is very fast and Eqs. (19) and (32) are not verified, there is still a normal solution given by Eq. (35). Nevertheless, one can not assure in this case that the normal solution approaches the equilibrium curve as $T \rightarrow \infty$.

To illustrate the above results we present in Fig. 1 three numerical solutions of the master equation for the TLS, Eq. (27), with asymmetry $\mu=0.5$. The variation of the temperature is

$$
\frac{d \gamma}{d t}=r \gamma
$$

with $r=10^{-4}$. The system was initially at equilibrium at three different temperatures, corresponding to $\gamma=10^{-10}$, $10^{-8}$, and $10^{-5}$. Also plotted are the equilibrium distri-

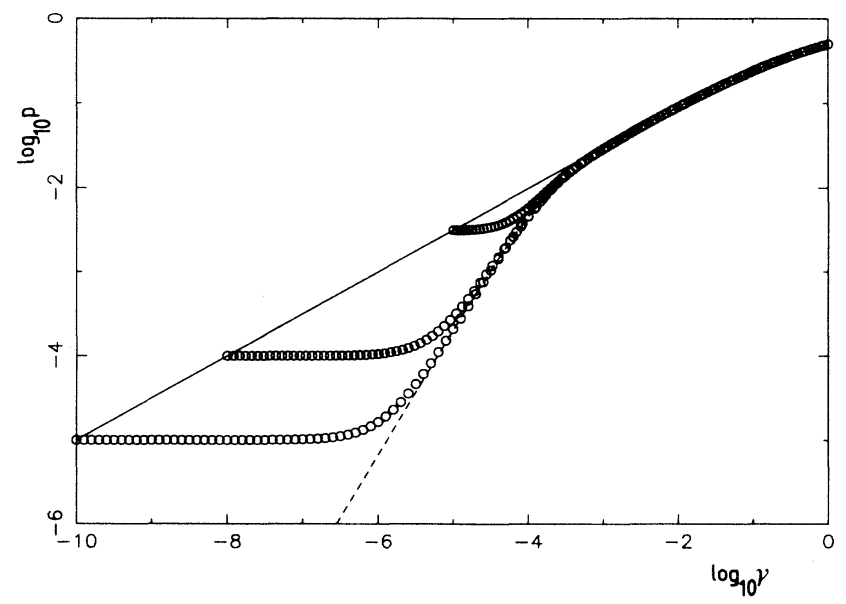

FIG. 1. Evolution of the upper-level population of the TLS as a function of the transition probability $\gamma$ for the heating law given by Eq. (37). The three curves correspond to $r=10^{-4}$ and $\mu=0.5$, with different initial conditions for the transition probability (from bottom to top $\gamma_{0}$ is equal to $10^{-10}, 10^{-8}$, and $10^{-5}$, respectively). The continuous line is the equilibrium population, and the dashed one is the normal solution Eq. (36). 


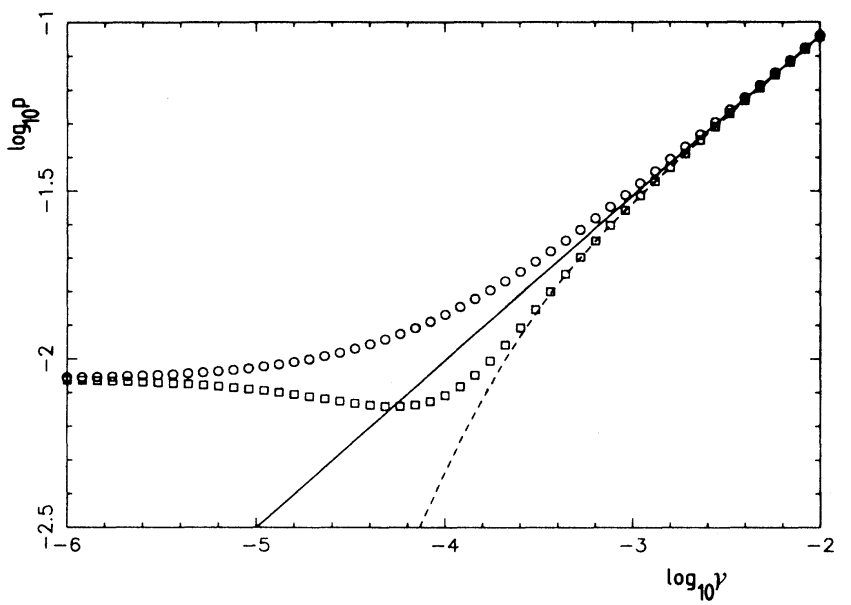

FIG. 2. Hysteresis cycle of the upper-level population when the TLS is first cooled (circles) and then heated (squares). The asymmetry is $\mu=0.5$ and the law of variation of $\gamma$ is Eq. (37), with $|r|=10^{-4}$ for both cooling and heating. The continuous line is the equilibrium population, and the dashed line is the normal solution.

bution and the normal solution Eq. (35). It is seen that at low temperatures there is a clear distinction between the equilibrium curve and the normal-solution curve, but both converge at high temperatures.

The figures also clearly shows one of the main points of this paper, namely, that the curves describing the heating of the system from an arbitrary initial condition tend to- ward the normal solution, and not towards the equilibrium curve. Therefore, if the system is heated from a point to the left of the equilibrium curve it will cross this curve, trying to reach the normal state. When the TLS is cooled down from equilibrium, it deviates from it in such a way that the value of $p(t)$ becomes greater than the equilibrium one $p_{0}(t)$, i.e., the cooling curve is to the left of the equilibrium curve [5-7]. If the system is then heated at the same rate it was cooled, it will not follow the same way back to equilibrium, but we know that it will approach the normal curve. This is the origin of the hysteresis that has been found numerically several times $[6,7]$. In Fig. 2 we show an example corresponding to the law given by Eq. (37). The circles correspond to the cooling process [Eq. (29) with $r<0$ ] and the squares to the heating one $(r>0)$. In both cases $|r|=10^{-4}$. Since the energy of the TLS is proportional to $p$, the different shape of the cooling and heating curves shows up clearly if one studies the behavior of the apparent heat capacity. While it monotonically decreases during the cooling, it presents a maximum along the heating path. This is a consequence of the fact that the equilibrium distribution has a greater average energy than the normal solution at the same temperature. In a forthcoming paper, the results obtained here will be applied to other systems, and their physical implications will be discussed in detail.

\section{ACKNOWLEDGMENT}

Partial support from the Dirección General de Investigación Científica y Técnica (Spain) through Grant No. PB89-0618 is gratefully acknowledged.
[1] S. A. Brawer, J. Chem. Phys. 81, 954 (1984); G. H. Frederickson and H. C. Andersen, Phys. Rev. Lett. 53, 1244 (1984); I. A. Weber and F. H. Stillinger, Phys. Rev. B 36, 7043 (1987); R. Schilling, J. Stat. Phys. 53, 1277 (1988); J. J. Brey and M. J. Ruiz-Montero, Phys. Rev. B 43, 585 (1991).

[2] N. G. van Kampen, Stochastic Processes in Physics and Chemistry (North-Holland, Amsterdam, 1981).

[3] G. W. Scherer, Relaxation in Glass and Composites (Wiley, New York, 1986).
[4] W. A. Phillips, J. Low Temp. Phys. 7, 351 (1972); P. Anderson, B. Halperin, and C. Varma, Philos. Mag. 25, 1 (1972).

[5] D. A. Huse and D. S. Fisher, Phys. Rev. Lett. 57, 2203 (1986); S. A. Langer and J. P. Sethna, ibid. 61, 570 (1988); J. J. Brey and A. Prados, Phys. Rev. B 43, 8350 (1991).

[6] S. A. Langer, J. P. Sethna, and E. R. Grannan, Phys. Rev. B 41, 2261 (1990).

[7] H. Szwarc, J. Non-Cryst. Solids 131-133, 252 (1991). 